\title{
Transsacrococcygeal approach to ganglion impar: radiofrequency application for the treatment of chronic intractable coccydynia
}

This article was published in the following Dove Press journal:

Journal of Pain Research

7 December 2016

Number of times this article has been viewed

\author{
Cemil Adas' \\ Ugur Ozdemir ${ }^{2}$ \\ Huseyin Toman ${ }^{3}$ \\ Nurettin Luleci ${ }^{4}$ \\ Emel Luleci ${ }^{5}$ \\ Hilal Adas ${ }^{6}$
}

'Anesthesiology and Reanimation Clinic, Șișli Hamidiye Etfal Education and Research Hospital, Istanbul, ${ }^{2}$ Department of Anesthesiology and Reanimation, Faculty of Medicine, Șifa University, Izmir, ${ }^{3}$ Department of Anesthesiology and Reanimation, Faculty of Medicine, Canakkale Onsekiz Mart University, Canakkale, ${ }^{4}$ Department of Anesthesiology and Reanimation, Faculty of Medicine, Algology Clinic, Maltepe University, ${ }^{5}$ Department of Health Education, Faculty of Health Education, Marmara University, ${ }^{6}$ Anesthesiology and Reanimation Clinic, Dr. Siyami Ersek Thoracic and Cardiovascular Surgery Training and Research Hospital, Istanbul, Turkey
Correspondence: Huseyin Toman Department of Anesthesiology and Reanimation, Faculty of Medicine, Canakkale Onsekiz Mart University, Kepez, Canakkale 17II0, Turkey Tel +90505 4505956

Email huseyintoman0I@hotmail.com
Objective: Coccydynia is defined as pain in the coccygeal region. Among the many causes of coccydynia, the most common cause is trauma as a result of falling on the buttocks, repetitive microtrauma, or childbirth. Several methods are currently used for the treatment of coccydynia, including nonsteroidal anti-inflammatory drugs, intrarectal manipulation, epidural injections, ganglion impar blocks, and radiofrequency treatment (RFT). Wemm and Saberski used the transacrococcygeal methods to reduce tissue trauma. RFT is a percutaneous minimally invasive procedure. In this study, we aimed to assess the effect of the transsacrococcygeal approach on ganglion impar RFT in patients with chronic coccydynia.

Methods: We retrospectively examined the data of 41 patients at the Department of Anesthesiology and Reanimation, Faculty of Medicine, Maltepe University (Pain Clinic), between January 1, 2010, and December 31, 2012.

Results: The mean age of the patients was $46.68 \pm 11.00$ years (range 28-67 [46] years). The average pain duration was $3.10 \pm 1.37$ years. The difference between visual analog scale scores of the preand postprocedure was statistically significant. In the examinations carried out in the sixth month of the treatment, $90.2 \%$ of patients had a successful outcome, whereas treatment failed in $9.8 \%$ of patients. According to our patients' data, most of them had pain due to a trauma, were female, and overweight. Visual analog scale difference between preprocedure and early postprocedure, preprocedure and first month, preprocedure and sixth month were statistically significant $(P=0.001)$. Conclusion: Based on the lower pain scores and low complication rates after the operations, the results suggest that application of RFT on ganglion impar by the transsacrococcygeal approach is an effective and safe method for the treatment of chronic coccydynia. Patient selection, technique, and experience are the most important factors affecting the success of this method.

Keywords: coccydynia, transsacrococcygeal approach, radiofrequency

\section{Introduction}

Coccydynia is a condition that is defined by pain in the coccyx, which is the inferior region of the vertebral column, comprising approximately four rudimentary vertebrae that articulate with the sacrum. Moreover, the coccyx has a close anatomical relationship with the sacral nerve roots and terminal end of the sympathetic chain called the ganglion impar. ${ }^{1,2}$ The ganglion impar (also known as the ganglion of Walther) is a solitary anatomical feature that is found on the ventral surface of the sacrococcygeal joint and provides nociceptive and sympathetic innervation to the perineal and anal areas. ${ }^{3,4}$

Coccydynia is a rare condition of various etiologies, affecting females five times more than males and is also associated with obesity. ${ }^{1,5}$ Trauma is the most common 
etiological cause of coccydynia as a result of falling on the buttocks, repetitive microtrauma, or childbirth. ${ }^{5,6}$ Coccydynia is diagnosed on the basis of a patient's history and clinical examination. Coccyx pain is typically provoked by sitting, ${ }^{6}$ and pain may present during rectal examination. ${ }^{2}$

Several methods are currently used for treating coccydynia, including nonsteroidal anti-inflammatory drugs, intrarectal manipulation, epidural injections, ganglion impar blocks, and radiofrequency treatment (RFT). ${ }^{1}$ For patients who do not respond to these forms of intervention, coccygectomy is often recommended. ${ }^{7}$

Wemm and Saberski used the transacrococcygeal method to reduce tissue trauma. RFT is a percutaneous minimally invasive procedure.

This study aimed to highlight the transsacrococcygeal approach to ganglion impar and the use of RFT for treating chronic intractable coccydynia.

\section{Methods}

Forty-one consecutive patients with chronic intractable perianal pain of various etiologies, who were treated with ganglion impar RFT, were included in our study. Fifty patients who were candidates for RFT first underwent a temporary ganglion impar block with $2 \mathrm{~mL}$ of $0.5 \%$ bupivacaine. RFT was offered to patients who had at least $50 \%$ reduction in pain according to their VAS score after local anesthesia. Nine patients who did not experience at least $50 \%$ reduction in pain according to their VAS score after local anesthesia were excluded. A transsacrococcygeal approach was used for both diagnostic and therapeutic interventions.

In our pain treatment center, we routinely obtain written informed consent from all our patients before starting the procedure in accordance with the Declaration of Helsinki and record pre- and postprocedure VAS scores. This protocol was reviewed and approved by the Şişli Hamidiye Etfal Education and Research Hospital of Medicine Institutional Review Board and the General Clinical Research Center (GCRC) protocol committee (Ünsal A), and patient consent was obtained from all patients for publishing this study.

The patient was placed in the prone position on the C-arm fluoroscopy table, with a cushion placed beneath the lower abdomen. After cleaning with an aseptic solution, a needle entry location was confirmed using fluoroscopy in an anterolateral position. Then, under the guidance of fluoroscopy in the lateral position, the needle was advanced to the sacrococcygeal disk. After passing to the disk, $1 \mathrm{~mL}$ of radiopaque dye was injected to verify the retroperitoneal field. We observed a "reverse comma" image when laterally

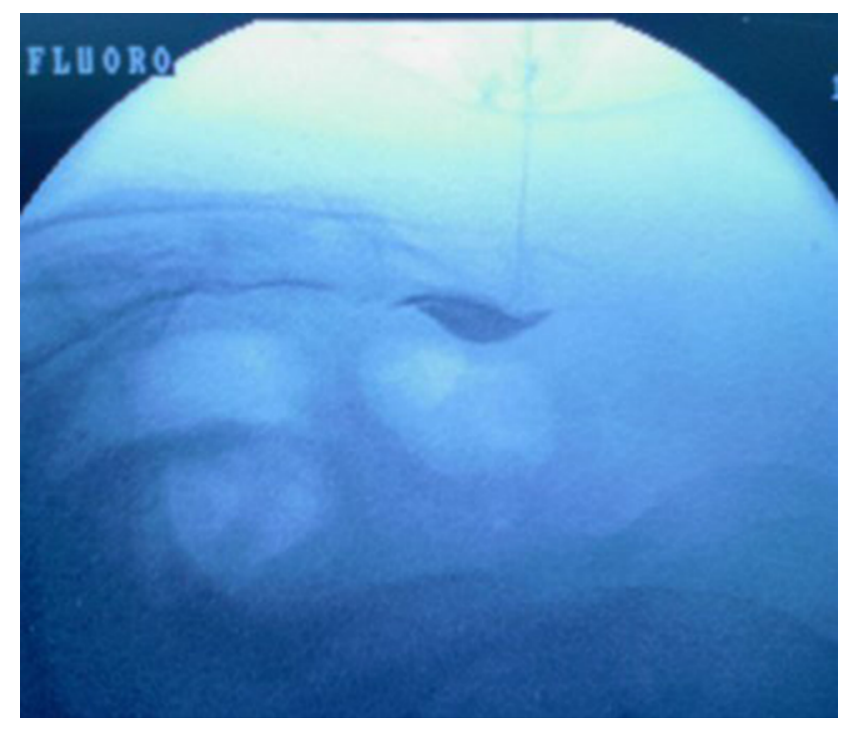

Figure I Lateral view and reverse comma image, transsacrococcygeal approach under fluoroscopy.

viewed (Figure 1). After confirming the location of the needle tip, $2 \mathrm{~mL}$ of $0.5 \%$ bupivacaine was injected. The VAS scores were noted at 30 minutes after the block, and RFT was planned if the patient's pain reduction was at least $50 \%$.

The candidate patients for RFT were informed before they were sent home, so that when the pain recurs, they should contact our headquarters for RFT. We applied RFT on patients who experienced temporary benefits with the administration of local anesthesia and at our center have references again.

Ganglion impar RFT was performed following written informed consent in accordance with the Declaration of Helsinki. The patient was placed in the prone position on the X-ray table with a pillow beneath the lower abdomen. After cleaning the skin for C-arm fluoroscopy, an entrance point was marked on the skin surface. Local anesthesia was administered at the site of the needle insertion. According to the guidance of the $\mathrm{C}$-arm fluoroscopy in the lateral position, a $22 \mathrm{G}, 10 \mathrm{~cm}$ needle was inserted through the skin to pierce the dorsal saccrococcygeal ligament at the midline.

The needle was then advanced through the vertebral disk until the tip was placed anterior to the ventral sacrococcygeal ligament, which was felt as the loss of resistance. The position of the needle tip was confirmed by injecting $1 \mathrm{~mL}$ of radiopaque dye in the retroperitoneal space. The spread of the dye provides a reverse comma appearance when observed in a lateral view (Figure 1).

After confirming the placement of the needle tip, RFT was performed at $80^{\circ} \mathrm{C}$ for 90 seconds, which is a conventional method for nerve ganglion ablation. During the procedure, we continuously communicated with the patient to monitor for complications (Figure 2). The total duration of the procedure 


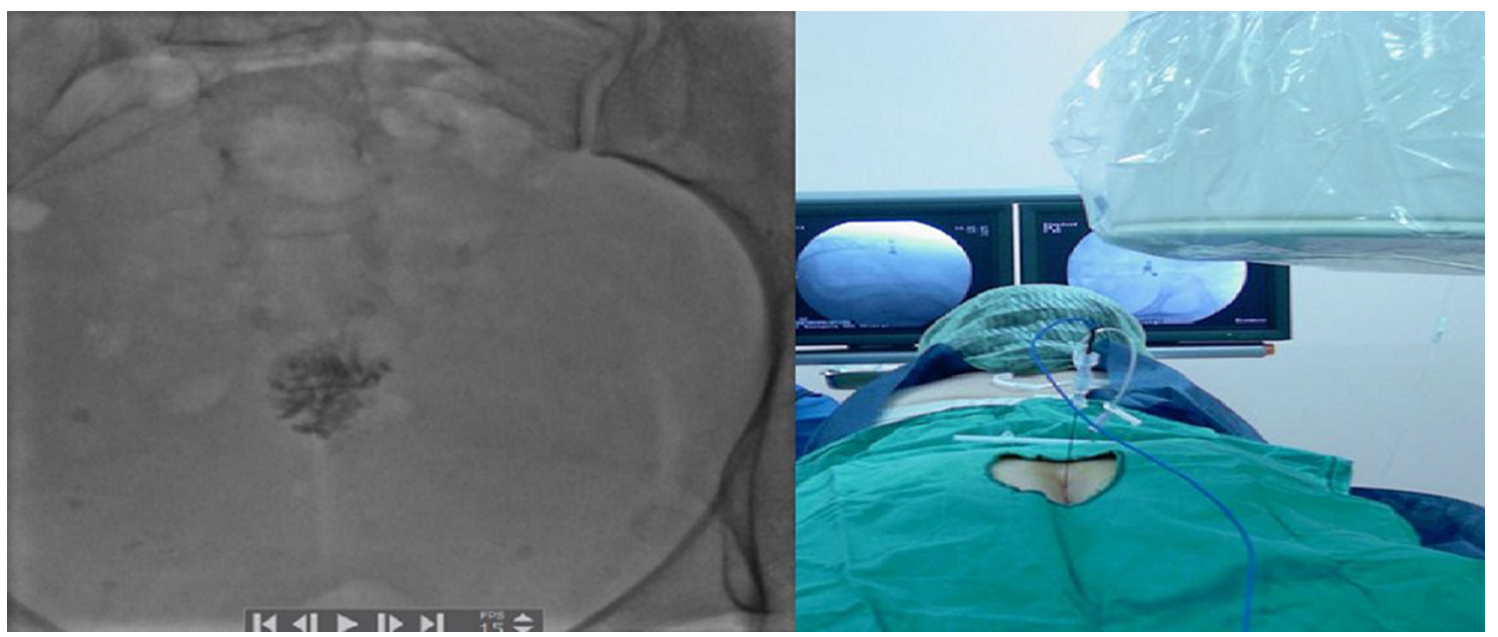

Figure 2 Anterioposterior view and patient position.

was 5 minutes. The VAS scores were noted at 2 hours and 6 hours after the procedure. The VAS score was noted by telephone conversation at 12 hours and 24 hours after the procedure. The patient was regularly followed up for the next 2 months. The patient was informed to report at any time if there was a pain with a VAS score of $\geq 5$. After 6 months, during the patients' clinical examination, we noted the VAS scores.

\section{Results \\ Statistical analyses}

Number Cruncher Statistical System 2007 (Kaysville, UT, USA) was used for statistical analyses. Data were analyzed using descriptive statistical methods (mean, SD, median, and frequency and rate). To compare data that were not normally distributed, Mann-Whitney $U$ test was performed. To compare qualitative data, a Fisher-Freeman-Halton test was performed. To evaluate the relationships between variables, Spearman's correlation analysis was performed. Results within 95\% confidence interval and with a $P$-value of $<0.05$ were assessed.

We retrospectively analyzed the collected data of 41 patients $(70.7 \%$ [ $n=29]$ females and $29.3 \%$ [ $=12]$ males $)$ with chronic coccydynia (pain for $>1$ year) who were treated by RFT of the ganglion impar in our Pain Clinic between January 1, 2012, and December 31, 2014.

The mean age of the patients was $46.68 \pm 11.00$ years (range 28-67 [46] years). The average pain duration was $3.10 \pm 1.37$ years. Statistically significant differences were observed between the pre- and postprocedure VAS scores. The last evaluation after the treatment (at 6 months) revealed that $90.2 \%$ of patients had a successful outcome and $9.8 \%$ of patients were deemed failures. According to our patient data, the etiological cause for most patients was trauma and most of them were females and overweight (Table 1). Malignancy was the risk factor for the failure of procedure. However, sex was not a risk factor for treatment failure (Table 2). The VAS scores changed over time; the difference in the VAS scores between preprocedure and at all subsequent time points was statistically significant ( $P=0.001)$ (Table 3 and Figure 3 ).

There were no statistically significant differences between the pre-early VAS, pre-1 month VAS, and pre-6 months VAS scores $(P>0.05)$. Although failure-accepted patients (VAS score $\geq 4$ ) mostly belonged to the malignant group, there were no significant differences between the decline ratio of the VAS scores of the benign and malignant groups $(6.17$ and 6.60, respectively) (Table 3). The fact that the initial pain scores were very high in the malignant group could have been a factor affecting our success.

All four cases considered as failures belonged to the normal body mass index group, and most of them were included in the malignant group. Although obesity is a risk factor for coccydynia, it does not affect the success rate of RFT treatment (Table 4).

There were no statistically significant differences between the pre-early VAS, pre- 1 month VAS, and pre- 6 months VAS scores with respect to age and pain duration $(P>0.05)$.

\section{Discussion}

A ganglion impar block can be used to treat acute or chronic perineal pain. ${ }^{8,9}$ Various methods exist to block the ganglion impar, such as local anesthetics, concomitant use of local anesthetics and steroids, alcohol or phenol, and neurolysis by RFT. ${ }^{10}$

We performed a block of the ganglion impar using RFT, which is a percutaneous minimally invasive procedure. Because of its widespread use and availability, we consider it as a good option for the safe destruction of the ganglion impar. ${ }^{10,11}$ 
Table I Patient description

\begin{tabular}{lll}
\hline Patient description & $\mathbf{n}$ & $\%$ \\
\hline Sex & & \\
$\quad$ Female & 29 & 70.7 \\
$\quad$ Male & 12 & 29.3 \\
Etiology & & \\
$\quad$ Malign causes & & \\
$\quad$ Malign causes (total) & 5 & 12.2 \\
$\quad$ Cervix CA & 2 & 4.9 \\
$\quad$ Rectum CA & 2 & 4.9 \\
$\quad$ Prostate CA & 1 & 2.4 \\
Benign causes & & \\
$\quad$ Benign causes (total) & 36 & 87.8 \\
$\quad$ Child Birth & 3 & 7.3 \\
$\quad$ Idiopathic & 12 & 29.3 \\
$\quad$ Trauma & 21 & 51.2 \\
BMI level & & \\
Normal & 11 & 26.8 \\
Overweight & 20 & 48.8 \\
Obesity & 10 & 24.4 \\
Procedure success & & \\
Successful (VAS $<4)$ & 37 & 90.2 \\
Failure (VAS $\geq 4)$ & 4 & 9.8 \\
\hline & Min-max (median) & Average \pm SD \\
\hline Age (year) & $28-67(46)$ & $46.68 \pm 11.00$ \\
BMI (kg/m ${ }^{2}$ ) & $17-34(27)$ & $26.46 \pm 4.12$ \\
Pain duration (year) & $1-6(3)$ & $3.10 \pm 1.37$ \\
\hline Abbrevitions: BMI & & \\
\hline
\end{tabular}

Abbreviations: $\mathrm{BMI}$, body mass index; $\mathrm{CA}$, cancer; max, maximum; min, minimum; SD, standard deviation; VAS, visual analog scale.

Table 2 Success rate related with malignite and sex

\begin{tabular}{llll}
\hline & Malignite & P-value \\
\cline { 2 - 3 } & Benign $(\mathbf{n = 3 6 )}$ & Malign (n=5) & \\
\hline $\begin{array}{l}\text { Success rate, n (\%) } \\
\text { Successful }\end{array}$ & $35(97.2)$ & $2(40.0)$ & $0.004^{*}$ \\
$\begin{array}{l}\text { Failure } \\
\text { Sex, } \mathrm{n}(\%)\end{array}$ & $1(2.8)$ & $3(60.0)$ & \\
$\begin{array}{l}\text { Female } \\
\text { Male }\end{array}$ & $26(72.2)$ & $3(60.0)$ & 0.620 \\
\hline
\end{tabular}

Note: Fisher-Freeman-Halton test: ${ }^{*} P<0.01$.

Table 3 VAS difference according to malignite

\begin{tabular}{|c|c|c|c|}
\hline \multirow[t]{2}{*}{ VAS difference } & \multicolumn{2}{|l|}{ Malignite } & \multirow[t]{2}{*}{$P$-value } \\
\hline & Benign & Malign & \\
\hline \multicolumn{4}{|l|}{ Pre-early VAS } \\
\hline Average \pm SD & $6.17 \pm 0.51$ & $6.60 \pm 0.55$ & 0.078 \\
\hline Min-max (median) & 4-6 (5) & 6-7 (7) & \\
\hline \multicolumn{4}{|l|}{ Pre-I month VAS } \\
\hline Average \pm SD & $5.39 \pm 0.60$ & $5.20 \pm 0.45$ & 0.415 \\
\hline Min-max (median) & $4-6(5)$ & $5-6(5)$ & \\
\hline \multicolumn{4}{|l|}{ Pre- 6 months VAS } \\
\hline Average \pm SD & $5.08 \pm 0.55$ & $4.80 \pm 0.45$ & 0.273 \\
\hline Min-max (median) & 4-6 (5) & $4-5(5)$ & \\
\hline
\end{tabular}

Note: Mann-Whitney $U$ test.

Abbreviations: max, maximum; min, minimum; VAS, visual analog scale; SD, standard deviation.

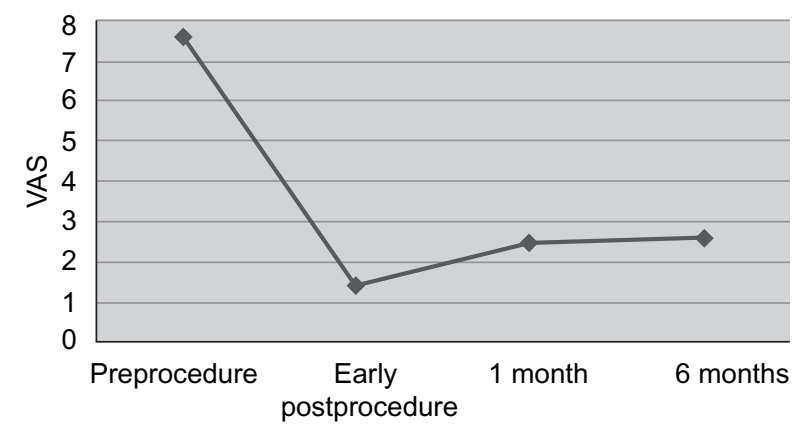

Figure 3 VAS change according to time.

Abbreviation: VAS, visual analog scale.

Table 4 BMI-related procedure success

\begin{tabular}{llll}
\hline & Procedure & P-value \\
\cline { 2 - 3 } & Successful, n (\%) & Failure, n (\%) & \\
\hline BMI & $7(18.9)$ & $4(100)$ & $0.006 *$ \\
Normal & $20(54.1)$ & $0(0)$ & \\
Overweight & $10(27)$ & $0(0)$ & \\
Obesity &
\end{tabular}

Notes: Fisher-Freeman-Halton test: ${ }^{* P}<0.01$. Normal BMI: $20-25 \mathrm{~kg} / \mathrm{m}^{2}$; Overweight BMI: $25-30 \mathrm{~kg} / \mathrm{m}^{2}$; Obesity: $>30 \mathrm{~kg} / \mathrm{m}^{2}$.

Abbreviation: BMI, body mass index.

The effectiveness of the treatment in patients with chronic coccydynia corresponded with a change in the VAS score. ${ }^{6,12-16}$ This condition affects females five times more than males. ${ }^{1,6}$ The most common cause of coccydynia is trauma as a result of falling on the buttocks. ${ }^{5,6}$ In a similar manner, our patients were mostly females and had a trauma history. We measured the treatment effectiveness by evaluating the changes in the VAS scores.

Patt and Plancarte ${ }^{17}$ first described a technique to block the ganglion impar. This conventional transanococcygeal membrane method is technically difficult, involves the risk of injuring the rectum and blood vessels, and has a high degree $(\sim 20 \%-30 \%)$ of failure. ${ }^{9}$ In addition, rectal perforation can cause contamination of the needle and increase the risk of needle stick injury to the surgeon's finger in the rectum. The transsacrococcygeal approach to the ganglion, described by Wemm and Saberski, is technically feasible and easy to learn and perform. ${ }^{8,9}$ Furthermore, Reig et al demonstrated that the transsacrococcygeal approach is technically feasible for RFT of the ganglion impar. ${ }^{10}$

We recommend using the transsacrococcygeal technique in our patients for RFT of the ganglion impar. Larger studies with randomized control groups would improve the level of evidence supporting the findings in this retrospective observational study.

Although the sacrococcygeal method is a safe and successful method, experience is as important for this method 
as for any other application. The fact that our entire operation was conducted by an experienced algologist could be an important factor that increased our chances of success. Multicenter-randomized studies can be prospectively conducted to verify this.

Larger population studies can be conducted using other treatment methods, such as nonsteroidal anti-inflammatory drugs, intrarectal manipulation, epidural injections, and blocking the ganglion impar with local anesthetics, as control groups to understand whether RFT is a truly effective treatment for these patients.

Coccydynia patients attempt various treatment methods before they are diagnosed. This situation prolongs the process until treatment. The effectiveness of the treatment using RFT compared with the period of pain before treatment can be investigated in subsequent studies. In our study, although the period of pain before treatment for all patients was recorded, we did not create groups according to pain duration and did not examine the treatment efficacy between the groups. To perform such studies, a larger population may be required.

Transsacrococcygeal RFT is a method that can be easily applied, has few complications, and can be repeated. Because transsacrococcygeal RFT is a minimally invasive treatment option, it should be considered before surgically treating patients with chronic coccydynia.

\section{Acknowledgment}

The authors have no financial relationship with the companies that manufactured the materials used in this study.

\section{Disclosure}

The authors report no conflicts of interest in this work.

\section{References}

1. Nathan ST, Fisher BE, Roberts CS. Coccydynia: a review of pathoanatomy, aetiology, treatment and outcome. J Bone Joint Surg Br. 2010;92(12):1622-1627.
2. Kerr EE, Benson D, Schrot RJ. Coccygectomy for chronic refractory coccygodynia: clinical case series and literature rewiew. J Neurosurg Spine. 2011;14(5):654-663.

3. Malec MM, Horosz B, Koleda I, et al. Neurolytic block of ganglion of Walther fort he management of chronic pelvic pain. Wideochir Inne Tech Maloinwazyjne. 2014;9(3):458-462.

4. Sagir A, Ozaslan S, Koroglu A. Application of ganglion impar block in patient with coccyx dislocation. Agri. 2011;23(3):129-133.

5. Maigne J, Doursounian L, Chatellier G. Causes and mechanisms of common coccydynia: role of body mass index and coccygeal trauma. Spine (Phila Pa 1976). 2000;25(23):3072-3079.

6. Howard PD, Dolan AN, Falco AN, Holland BM, Wikinson CF, Zink AM A comparison of conservative interventions and their effectiveness for coccydynia: a systematic review. J Man Manip Ther. 2013;21(4): 213-219.

7. Karadimos EJ, Trypsiamis G, Giannoudis PV. Surgical treatment of coccygodynia: an analytic review of the literature. Eur Spine J. 2011; 20(5):698-705.

8. Wemm K Jr, Saberski L. Modified approach to block the ganglion impar (Ganglion of Walther). Reg Anesth. 1995;20(6):544.

9. Toshniwal GR, Dureja GP, Prashanth SM. Transsacrococcygeal approach to ganglion impar block for management of chronic perineal pain: a prospective observational study. Pain Physician. 2007;10(5): 661-666.

10. Reig E, Abejon D, del Pozo C, Insausti J, Contreas R. Thermocoagulation of the ganglion impar or ganglion of Walther: description of a modified approach. Preliminary result in chronic, nononcological pain. Pain Pract. 2005;5(2):103-110.

11. Demircay A, Kabatas S, Cansever T, Yilmaz C, Tuncay C, Altınors N. Radiofrequency thermocoagulation of ganglion impar in the management of coccydynia: preliminary results. Turk Neurosurg. 2010;20(3): 328-333.

12. Maigne J, Chatellier G, Le Faou M, Archambeau M. The treatment of chronic coccydynia with intrarectal manipulation: a random controlled study. Spine (Phila Pa 1976). 2006;31(18):E621-E627.

13. Khatri SM, Nitsure P, Jatti RS. Effectiveness of coccygeal manipulation in coccydynia: a randomized control trial. Indian J Physiother Occup Ther. 2011;5:110-112.

14. Datir A, Connell D. CT-guided injection for ganglion impar blockade: a radiological approach to the management of coccydynia. Clin Radiol. 2010;65(1):21-25.

15. Khan SA, Kumar A, Varshney MK, Trikha V, Yadav CS. Dextrose prolotherapy for recalcitrant coccygodynia. J Orthop Surg (Hong Kong). 2008;16(1):27-29.

16. Mitra R, Cheung L, Perry P. Efficacy of fluoroscopically guided steroid injections in the management of coccydynia. Pain Physician. 2007;10(6): 775-778.

17. Patt RB, Plancarte R. In: Waldman SD, Winnie AP, editors. Superior Hypogastric Plexus Block, Interventional Pain Management. Philadelphia, PA: WB Saun-ders; 1996:384-391.
Journal of Pain Research

\section{Publish your work in this journal}

The Journal of Pain Research is an international, peer reviewed, open access, online journal that welcomes laboratory and clinical findings in the fields of pain research and the prevention and management of pain. Original research, reviews, symposium reports, hypothesis formation and commentaries are all considered for publication.

\section{Dovepress}

The manuscript management system is completely online and includes a very quick and fair peer-review system, which is all easy to use. Visit http://www.dovepress.com/testimonials.php to read real quotes from published authors. 